\title{
Exenatide decreases hepatic fibroblast growth factor 21 resistance in non-alcoholic fatty liver disease in a mouse model of obesity and in a randomised controlled trial
}

\author{
S. L. Samson • P. Sathyanarayana • M. Jogi • \\ E. V. Gonzalez • A. Gutierrez • R. Krishnamurthy • \\ R. Muthupillai $\cdot$ L. Chan $\cdot$ M. Bajaj
}

Received: 12 June 2011 / Accepted: 26 August 2011 /Published online: 29 September 2011

(C) Springer-Verlag 2011

\begin{abstract}
Aims/hypothesis Systemic fibroblast growth factor (FGF)21 levels and hepatic FGF21 production are increased in nonalcoholic fatty liver disease patients, suggesting FGF21 resistance. We examined the effects of exenatide on FGF21 in patients with type 2 diabetes and in a diet-induced mouse model of obesity (DIO).

Methods Type 2 diabetes mellitus patients $(n=24)$ on diet and/or metformin were randomised (using a table of random numbers) to receive additional treatment consisting of pioglitazone $45 \mathrm{mg}$ /day or combined therapy with pioglitazone $(45 \mathrm{mg} /$ day $)$ and exenatide $(10 \mu \mathrm{g}$ twice daily) for 12 months in an open label parallel study at the Baylor Clinic. Results Twenty-one patients completed the entire study and were included in the analysis. Pioglitazone treatment $(n=10)$ reduced hepatic fat as assessed by magnetic resonance
\end{abstract}

S.L. Samson and P. Sathyanarayana contributed equally to this study.

Electronic supplementary material The online version of this article (doi:10.1007/s00125-011-2317-z) contains peer-reviewed but unedited supplementary material, which is available to authorised users.

S. L. Samson · P. Sathyanarayana • E. V. Gonzalez • A. Gutierrez •

L. Chan $\cdot$ M. Bajaj $(\bowtie)$

Diabetes and Endocrinology Research Center,

Endocrinology Division, Baylor College of Medicine,

1709 Dryden Street,

Houston, TX 77030, USA

e-mail: mandeepbajaj@hotmail.com

S. L. Samson · M. Jogi $\cdot$ A. Gutierrez $\cdot$ L. Chan $\cdot$ M. Bajaj Departments of Medicine, St Luke's Episcopal Hospital,

Houston, TX, USA

R. Krishnamurthy $\cdot$ R. Muthupillai

Department of Radiology, St Luke's Episcopal Hospital,

Houston, TX, USA spectroscopy, despite a significant increase in body weight ( $\Delta=3.7 \mathrm{~kg})$; plasma FGF21 levels did not change $(1.9 \pm 0.6$ to $2.2 \pm 0.6 \mathrm{ng} / \mathrm{ml}$ [mean $\pm \mathrm{SEM}]$ ). However, combined pioglitazone and exenatide therapy $(n=11)$ was associated with a significant reduction of FGF21 levels $(2.3 \pm 0.5$ to $1.1 \pm$ $0.3 \mathrm{ng} / \mathrm{ml}$ ) and a greater decrease in hepatic fat. Besides weight gain observed in the pioglitazone-treated patients, lower extremity oedema was observed as a side effect in two of the ten patients. Three patients who received pioglitazone and exenatide combination therapy complained of significant nausea that was self-limiting and did not require them to leave the study. In DIO mice, exendin- 4 for 4 weeks significantly reduced hepatic triacylglycerol content, decreased hepatic FGF21 protein and mRNA, and enhanced phosphorylation of hepatic AMP-activated protein kinase (AMPK) and acetyl-CoA carboxylase, although no significant difference in weight and body fat was observed. Hepatic FGF21 correlated inversely with hepatic AMPK phosphorylation

Conclusions/interpretation In type 2 diabetes mellitus, combined pioglitazone and exenatide therapy is associated with a reduction in plasma FGF21 levels, as well as a greater decrease in hepatic fat than that achieved with pioglitazone therapy. In DIO mice, exendin-4 treatment reduces hepatic triacylglycerol and FGF21 protein, and enhances hepatic AMPK phosphorylation, suggesting an improvement of hepatic FGF21 resistance.

Trial registration number: ClinicalTrials.gov NCT 01432405 Funding: American Diabetes Association, the Ron MacDonald Foundation at St Luke's Episcopal Hospital, Amylin Pharmaceuticals, Eli-Lilly, NIH Molecular Medicine Scholars Training Grant, NIH Diabetes and Endocrinology Research Center (DERC) at Baylor College of Medicine, and the T.T. and W.F. Chao Foundation. 
Keywords AMPK · Exenatide · FGF21 - GLP-1 · Hepatic steatosis $\cdot$ NAFLD $\cdot$ Pioglitazone

$\begin{array}{ll}\text { Abbreviations } \\ \text { ACC } & \text { Acetyl-CoA carboxylase } \\ \text { AMPK } & \text { AMP-activated protein kinase } \\ \text { DIO } & \text { Diet-induced mouse model of obesity } \\ \text { FGF } & \text { Fibroblast growth factor } \\ \text { FGFR } & \text { FGF receptor } \\ \text { GLP-1 } & \text { Glucagon like peptide-1 } \\ \text { GLP1R } & \text { GLP-1 receptor } \\ \text { HDAd } & \text { Helper-dependent adenoviral vector } \\ \text { HFD } & \text { High-fat diet } \\ \text { MRS } & \text { Magnetic resonance spectroscopy } \\ \text { NAFLD } & \text { Non-alcoholic fatty liver disease } \\ \text { PGC-1 } \alpha & \text { PPAR- } \gamma \text { coactivator } \\ \text { PPAR } & \text { Peroxisome proliferator-activated receptor } \\ \text { TBST } & \text { TRIS-buffered saline with } 0.05 \% \text { Tween } \\ \text { WAT } & \text { White adipose tissue }\end{array}$

\section{Introduction}

Fibroblast growth factors (FGFs) are a group of peptides that regulate diverse biological functions, including cell differentiation, growth and angiogenesis [1]. Recently, a subfamily of FGFs has been identified, whose members interact with nuclear receptors and which plays an important role in liver and adipose tissue metabolism. This subfamily includes FGF19, which regulates energy expenditure, and FGF21, which regulates glucose and lipid metabolism in fasting and ketotic states in animals [2,3]. In rodents, FGF21 is predominantly produced in the liver, where it enhances hepatic fat oxidation [3]. FGF21 also increases adipocyte insulin sensitivity and regulates lipolysis in white adipose tissue (WAT) [3]. In mouse models of obesity, FGF21 enhances insulin sensitivity, reduces triacylglycerol levels and hepatic steatosis, and improves glucose tolerance [4-6]. Recent work suggests that these changes may occur through the effects of FGF21 on AMP-activated protein kinase (AMPK) activity [7].

These observations have generated considerable interest in the potential of FGF21 as a treatment for type 2 diabetes. However, more recent studies have suggested that there is a state of 'FGF21 resistance' in obese and type 2 diabetic humans, as well as in rodent models of obesity [8]. Plasma FGF21 levels are increased in obese and type 2 diabetic patients, and correlate with hepatic and peripheral insulin resistance [9]. Non-alcoholic fatty liver disease (NAFLD) is common in type 2 diabetic patients and Dushay et al. [10] have recently shown that liver FGF21 protein levels and RNA are increased in association with hepatic steatosis in obese humans with NAFLD. Similarly, plasma FGF21 levels are also increased significantly in NAFLD. In mouse models of obesity, circulating FGF21 levels are elevated and FGF21 abundance is also increased in liver and WAT [8]. In these animal models, FGF21 signalling is also impaired in the liver and WAT. In addition, levels of FGF receptors (FGFRs), including FGFR1 in the liver, and FGFR2 and FGFR3 in WAT, are reduced in a diet-induced mouse model of obesity (DIO) [8]

Exenatide (exendin 4) is a glucagon like peptide-1 (GLP-1) receptor (GLP1R) agonist that enhances glucosedependent insulin secretion and suppression of inappropriately high glucagon secretion, leading to improved glycaemic control in patients with type 2 diabetes. Healthy non-diabetic participants, obese participants and patients with type 2 diabetes show a significantly reduced appetite and body weight following GLP1R agonist treatment [11, 12]. We and others $[13,14]$ have also demonstrated reduced hepatic triacylglycerol content in rodent models of obesity. In addition, the expression of key lipogenic genes is suppressed. More recently, we have shown that exenatide augments the reduction of hepatic fat and increase of circulating adiponectin levels effected by pioglitazone in patients with type 2 diabetes [15]. However, the effect of exenatide on circulating FGF21 levels in humans, as well as its relationship to hepatic steatosis, has not been previously studied.

The current study examined the effect of exenatide on hepatic FGF21 in patients with type 2 diabetes and NAFLD. We further explored this in a DIO, where continuous delivery of exendin- 4 for 4 weeks had positive effects on hepatic triacylglycerol content, plasma and liver FGF21 levels, and hepatic AMPK phosphorylation.

\section{Methods}

Human participants As previously described [15], 21 type 2 diabetes patients (age $52 \pm 3$ [mean \pm SEM] years, BMI $32.0 \pm 1.5 \mathrm{~kg} / \mathrm{m}^{2}, \mathrm{HbA}_{1 \mathrm{c}} 8.2 \pm 0.4 \%$ [66 mmol $\left./ \mathrm{mol}\right]$ ) on diet and/or metformin therapy completed the entire study. Briefly, the entry criteria included: age 30 to 70 years, stable body weight $( \pm 1 \mathrm{~kg})$ for at least 3 months before study and fasting plasma glucose 7.0 to $14.5 \mathrm{mmol} / \mathrm{l}$. Patients with alanine aminotransferase or aspartate aminotransferase greater than 2.5 times the upper limit of normal were excluded from the study. No participant engaged in any heavy exercise. Patients who had received insulin, sulfonylureas, sitagliptin, exenatide or a thiazolidinedione within the previous 3 months were excluded. Metformintreated diabetic patients were included, but the dosage of metformin was not altered throughout the duration of the study. The results of hepatic fat measurements and anthropometric/metabolic variables in these patients have 
been previously published [15]. The Institutional Review Board of the Baylor College of Medicine approved the protocol. All participants gave signed voluntary informed consent before participation.

Three weeks before the study, participants met a dietitian and were instructed to consume a weight-maintaining diet containing $50 \%$ carbohydrate, $30 \%$ fat and $20 \%$ protein. During the week prior to start of the study medication(s), all participants received baseline measurements of plasma FGF21 and measurement of liver fat content by magnetic resonance spectroscopy (MRS). All studies were started at 08:00 hours following a 10 to $12 \mathrm{~h}$ overnight fast. After completion of these studies, participants were randomised (using a table of random numbers) to participate in an openlabel study in one of the two treatment arms: (1) pioglitazone $30 \mathrm{mg}$ /day orally for 2 weeks followed by pioglitazone $45 \mathrm{mg}$ /day orally for 50 weeks $(n=10)$; or (2) exenatide $5 \mu \mathrm{g}$ injected subcutaneously twice daily and pioglitazone $30 \mathrm{mg}$ /day orally for 2 weeks, followed by exenatide $10 \mu \mathrm{g}$ subcutaneously twice daily and pioglitazone $45 \mathrm{mg} /$ day orally for 50 weeks $(n=11)$. Seventeen participants had been taking a stable dose of metformin for at least 3 months before study and four participants had been treated with diet alone. All participants underwent a repeat measurement of plasma FGF21 (drawn after a 10 to $12 \mathrm{~h}$ overnight fast) and determination of hepatic fat content by MRS at the end of 12 months.

Animals Animal experiments were approved by the Institutional Animal Care and Use Committee at Baylor College of Medicine and care of mice was humane according to the criteria outlined in the Guide for the Care and Use of Laboratory Animals. C57/BL6 male mice were from The Jackson Laboratory (Bar Harbor, ME, USA) and were housed in the Taub Animal Facility (Baylor College of Medicine, Houston, TX, USA). For the DIO, 14-week-old mice were fed a $60 \%$ high-fat diet (HFD; Harlan Laboratories, Houston, TX, USA) for 8 weeks. Exendin 4 treatment was started at 18 weeks of age, 4 weeks after initiation of the HFD. Treatment was by continuous delivery of exendin 4 for 28 days using a subcutaneous mini-osmotic pump (Alzet2004; Durect, Cupertino, CA, USA). Exendin 4 peptide (E7144; Sigma-Aldrich, St Louis, MO, USA) was solubilised in PBS at a concentration of $100 \mu \mathrm{g} / \mathrm{ml}$ with $0.1 \%$ mouse serum albumin (Sigma). The primed pumps were inserted subcutaneously in the dorsal area under anaesthesia. The control mice received PBS with $0.1 \%$ (wt/vol.) mouse serum albumin, also via pumps. Exendin 4 levels in plasma were measured by enzyme immunoassay (Phoenix Pharmaceuticals, Burlingame, CA, USA). Echo magnetic resonance imaging (Echo Medical System, Houston, TX, USA) was used for mouse body composition analysis.
Analytical determinations Mouse plasma was collected in heparinised tubes. Mouse insulin levels were measured using an ultrasensitive mouse insulin ELISA (Mercodia, Uppsala, Sweden). Plasma FGF21 concentration was measured by ELISA (BioVendor, Candler, NC, USA). This assay detects human and mouse FGF21, and does not crossreact with other members of the FGF family.

Western blotting Western blotting was used to measure FGF21 content, as well as AMPK and acetyl-CoA carboxylase (ACC) phosphorylation in the liver. The antibodies were as follows: FGF21 (detects mouse and human; Abcam, Cambridge, MA, USA); $\beta$-actin, phosphoAMPK $\alpha$ (Tyr172) and total AMPK $\alpha$, and phospho-ACC (Ser79) and total ACC1 (Cell Signaling Technology, Danvers, MA, USA). Liver samples were homogenised in ice-cold lysis buffer ( $20 \mathrm{mmol} / 1$ TRIS [pH 7.5], $10 \mathrm{mmol} /$ 1 sodium pyrophosphate, $100 \mathrm{mmol} / 1$ sodium fluoride, $2 \mathrm{mmol} / \mathrm{l}$ sodium orthovanadate, $5 \mathrm{mmol} / \mathrm{l}$ EDTA [pH 8.0], $1 \%$ Nonidet $\mathrm{P}-40,1 \mathrm{mmol} / 1$ phenylmethylsulfonyl fluoride, $3 \mathrm{mmol} / \mathrm{l}$ benzamidine, $10 \mu \mathrm{g} / \mathrm{ml}$ leupeptin and $10 \mu \mathrm{g} / \mathrm{ml}$ aprotinin). Homogenates were rotated for $1 \mathrm{~h}$ at $4^{\circ} \mathrm{C}$ and then centrifuged for $10 \mathrm{~min}$ at $14,000 \mathrm{~g}$ and $4^{\circ} \mathrm{C}$. The supernatant fractions were collected and protein concentrations measured by the Bradford assay. Lysate proteins were separated by SDS-PAGE (Bio-Rad Laboratories, Hercules, CA, USA). The order of loading of the samples from each particular animal was the same for each gel. After transfer of the proteins to nitrocellulose membrane (Bio-Rad), the membrane was blocked with TRISbuffered saline with $0.05 \%$ Tween (TBST) and $5 \%$ (wt/ vol.) non-fat dry milk, and then incubated overnight at $4{ }^{\circ} \mathrm{C}$ with 1:500 dilution of primary antibody in TBST with $5 \%$ $\mathrm{wt} / \mathrm{vol}$. bovine serum albumin. The next day the blot was washed with TBST and incubated with the detection antibody (horseradish peroxidase-conjugated goat antirabbit IgG, 1:10,000; Bio-Rad) at room temperature for $1 \mathrm{~h}$. Protein bands were detected by chemiluminescence (Super Signal West Pico; Thermo Scientific, Rockford, IL, USA) using autoradiographic film. The films were scanned and the bands quantified with the Image J program (http:// rsbweb.nih.gov/ij/, accessed 5 May 2009).

Glucose tolerance testing in mice Glucose tolerance was assessed as described [14] in mice fasted for $16 \mathrm{~h}$. Mice were injected intraperitoneally with $1.5 \mathrm{~g}$ glucose per $\mathrm{kg}$ body weight. Glucose levels were measured by glucometer (Lifescan, Milpitas, CA, USA) from tail blood. Plasma was also collected at each time point for measurement of insulin.

Hepatic fat content Hepatic fat content in patients with type 2 diabetes was measured by MRS as previously described [15-17]. In the animal studies, total lipid was extracted 
from liver by the Bligh-Dyer method [18] and the extracted triacylglycerol quantified by colorimetric assay (Thermo Scientific Infinity, Rockford, IL, USA).

Quantitative RT-PCR Total RNA was isolated from liver using a kit (Aurum Mini RNA Extraction; Bio-Rad) according to the manufacturer's instructions. RNA was subjected to DNaseI digestion (Invitrogen, Carlsbad, CA, USA) and then reverse transcription using a first strand synthesis system (Superscript III; Invitrogen) primed with oligo dT. The cDNAs were subjected to PCR as follows: one cycle at $95^{\circ} \mathrm{C}$ for $5 \mathrm{~min}, 40$ cycles at $95^{\circ} \mathrm{C}$ for $1 \mathrm{~min}$, $55^{\circ} \mathrm{C}$ for $1 \mathrm{~min}$ and $72^{\circ} \mathrm{C}$ for $1 \mathrm{~min}$, and one cycle at $72^{\circ} \mathrm{C}$ 10 min. Quantitative RT-PCR was performed with iQ SYBR Green Supermix (Bio-Rad) and Rox reference dye using a quantitative PCR machine (Mx3000P; Agilent Technologies, Santa Clara, CA, USA). Primers were designed using the Primer3 program (http://frodo.wi.mit. edu/primer3/input.htm, accessed 11 April 2011) to cross an intron, have a temperature of $58^{\circ} \mathrm{C}$ to $62^{\circ} \mathrm{C}$, and to produce an approximately $200 \mathrm{bp}$ product. Primers were synthesised by Sigma Genosys (Sigma-Aldrich) and are shown in electronic supplemental material Table 1.

Statistical analysis Comparisons between groups were made using Student's $t$ test and calculation of the Pearson product-moment correlation coefficient. Data are presented as mean \pm SEM.

\section{Results}

Effect of exenatide and pioglitazone treatment on hepatic fat content and fasting plasma FGF21 levels in patients with type 2 diabetes As previously described [15], type 2 diabetic patients on pioglitazone or combined pioglitazone and exenatide treatment had significantly reduced levels of fasting plasma triacylglycerol, glucose, NEFA and insulin. The pioglitazone-treated patients had increased body weight $(\Delta=3.7 \mathrm{~kg})$ over the 12 month treatment period, while patients receiving combined pioglitazone and exenatide therapy had no significant change in body weight $(\Delta=0.2 \mathrm{~kg})$. Furthermore, the reduction of hepatic fat content was significantly greater in patients on combined pioglitazone and exenatide therapy than in those on pioglitazone treatment alone ( $\Delta=61 \%$ vs $41 \%, p<0.05$; Fig. $1 \mathrm{a}, \mathrm{b})$.

Fasting plasma FGF21 levels did not change after 12 months of pioglitazone therapy $(1.9 \pm 0.6$ vs $2.2 \pm$ $0.6 \mathrm{ng} / \mathrm{ml}[$ mean \pm SEM]; Fig. 1c) despite the reduced hepatic fat content. However, the combination of pioglitazone and exenatide caused a significant decline in fasting plasma FGF21 after 12 months of treatment (from $2.3 \pm 0.5$ to $1.1 \pm 0.3 \mathrm{ng} / \mathrm{ml}, p<0.01$; Fig. $1 \mathrm{~d})$.
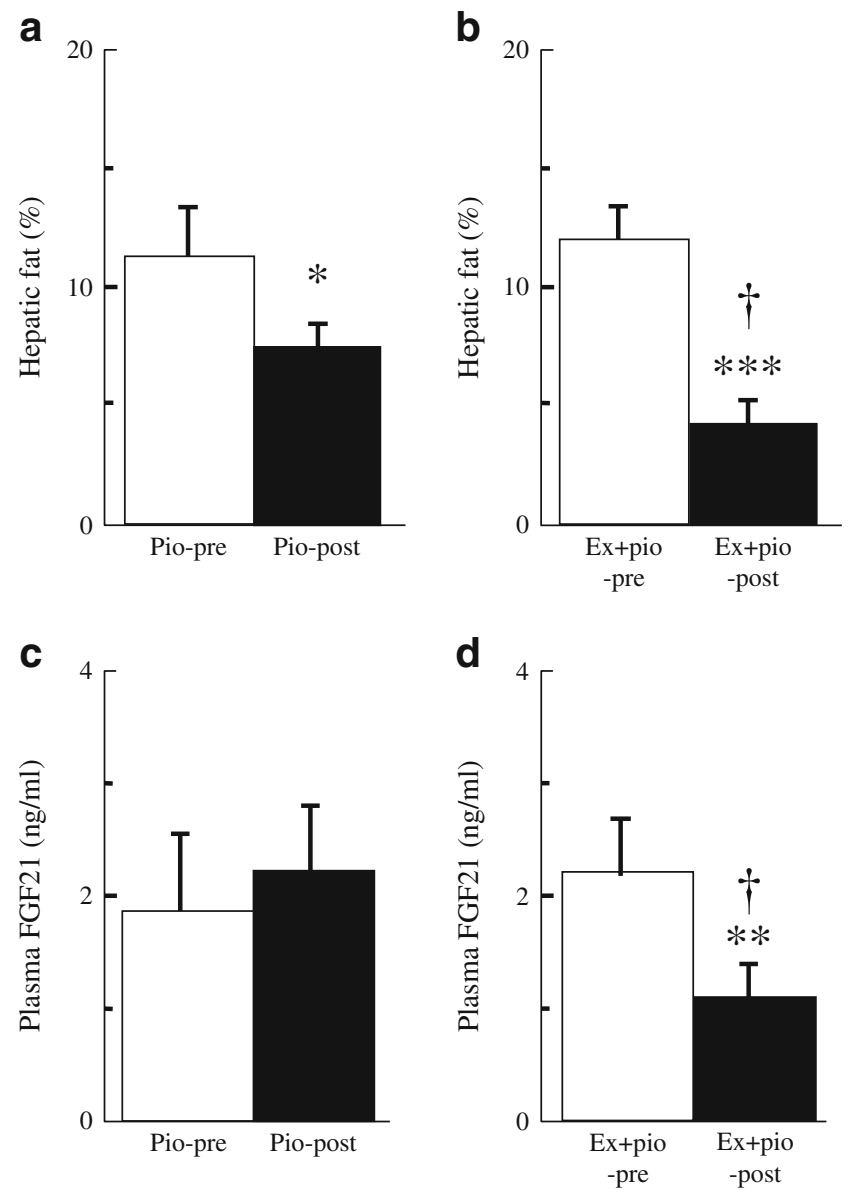

Fig. 1 Effect of pioglitazone (Pio) therapy $(n=10)$, and of combined exenatide and pioglitazone $(\mathrm{Ex}+$ pio $)$ therapy $(n=11)$ on hepatic fat content $(\mathbf{a}, \mathbf{b})$ and fasting plasma FGF21 levels $(\mathbf{c}, \mathbf{d})$ in patients with type 2 diabetes. ${ }^{*} p<0.05,{ }^{* *} p<0.01$ and ${ }^{* * *} p<0.001$ for pre-therapy vs post-therapy; ${ }^{\dagger} p<0.05$ for Ex+pio vs Pio

Effect of continuous delivery of exendin-4 on glucose tolerance and liver triacylglycerol content in DIO We previously observed decreased hepatic triacylglycerol content in HFD-induced obese (DIO) mice treated with a helper-dependent adenoviral vector (HDAd) for long-term (16 weeks) expression of exendin 4 [14]. We further used this HFD mouse model to help us understand the effects of exendin 4 treatment on hepatic steatosis and specifically FGF21 levels. For this study, we used a subcutaneous osmotic pump delivery system, which delivers an estimated $0.5 \mathrm{nmol}$ per day of exendin 4 , resulting in pharmacological plasma levels averaging $1,734 \pm 862 \mathrm{pmol} / 1 \quad(n=4)$ at 2 weeks post-implantation. For the short duration of the study (28 days), total body weight and fat mass did not change significantly between the control mice (HFD saline) and the exendin 4-treated mice (HFD exendin 4; Fig. 2a). However, the glucose tolerance test revealed markedly improved glucose clearance at 4 weeks of exendin 4 treatment while on HFD, confirming that the exendin 4 was biologically active (Fig. 2b). Absolute insulin levels 

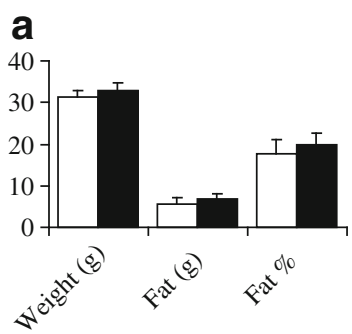
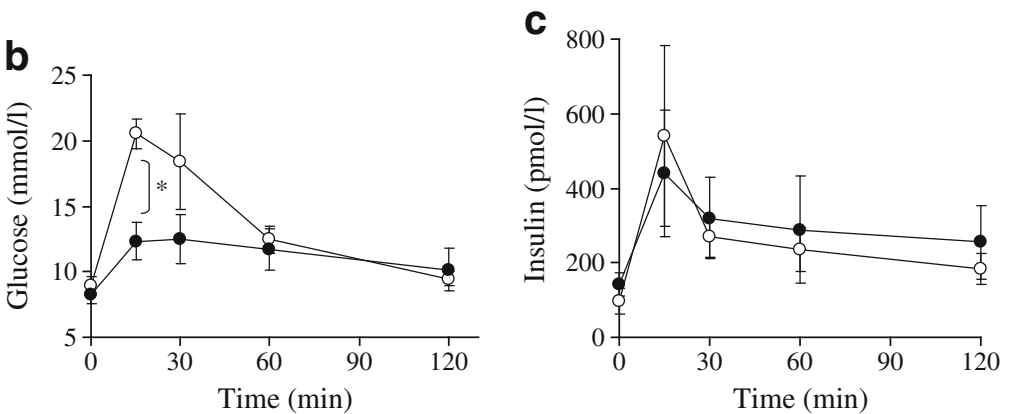

Fig. 2 a Mouse weight, fat (g) and per cent fat content after 4 weeks of mini-osmotic pump treatment while on HFD. b Glucose tolerance testing was performed after 4 weeks treatment. Fasted mice $(16 \mathrm{~h})$

during the glucose tolerance test were not augmented by exendin 4 compared with saline, which is contrary to the expected 'incretin' effect of the GLP-1 analogue (Fig. 2c). However, this is very consistent with our previous observations using vector delivery of exendin 4 [14] and we believe it reflects an improvement in insulin sensitivity and peripheral glucose disposal, which reduces the need for augmented insulin secretion.

With the evidence that the Alzet pump-delivered exendin 4 was functional, we examined its effect on hepatic steatosis (Fig. 3a). In the HFD exendin 4 mice, the absolute liver weight $(p=0.04)$ and liver weight as per cent of body weight $(p=0.006)$ were significantly decreased even though the body weights at death were not significantly different between groups $(30.5 \pm 1.4 \mathrm{~g}$ HFD saline vs $32.3 \pm 3.3 \mathrm{~g}$ HFD exendin $4 ; p=0.35$ ). Furthermore, the total triacylglycerol content per liver (Fig. 3c) or per g of liver (Fig. 3b) in the exendin 4 -treated mice was less than $50 \%$ of that of the control mice (HFD saline).

Continuous delivery of exendin 4 decreases hepatic Fgf21 expression and protein in DIO mice and increases hepatic AMPK phosphorylation Having demonstrated improvements in hepatic triacylglycerol content, we examined the were injected intraperitoneally with $1.5 \mathrm{mg} / \mathrm{kg}$ glucose. c Plasma insulin levels during the same glucose tolerance test. $n=4$ in each group, ${ }^{*} p<0.05$. White, saline control; black, exendin 4

levels of FGF21 in the plasma and liver. HFD-fed mice had more than threefold higher FGF21 levels in plasma compared with age-matched chow-fed mice (Fig. 4a). Treatment with exendin 4 resulted in lower FGF21 levels, which were not significantly different from non-obese chow fed mice (Fig. 4a). Treatment with exendin 4 also significantly reduced liver Fgf21 mRNA (Fig. 4b) and protein (Fig. 4c). Furthermore, liver FGF21 correlated with plasma FGF21 levels $(r=0.742, p=0.04$; Fig. $4 d)$, as well as with liver weight expressed as per cent of body weight $(r=0.838, p=0.01$; Fig. 4e). Liver triacylglycerol content $(\mathrm{mg} / \mathrm{g})$ tended to correlate with liver weight as a per cent of body weight $(r=0.705, p=0.07)$. Interestingly, FGF2 1 levels were not correlated with total body weight, body fat (g) or fat as per cent of total body weight. FGF21 has been shown to regulate AMPK phosphorylation and activity [7]. In our model of continuous exendin 4 delivery, there was a significant increase in AMPK phosphorylation in the liver, despite a reduction of hepatic and plasma FGF21 (Fig. 4f). Phosphorylation of the AMPK target, ACC, was also significantly increased in the exendin 4treated mice (Fig. 4f). AMPK phosphorylation correlated strongly and negatively $(r=-0.865, p=0.006)$ with hepatic FGF21, suggesting that exendin 4 treatment ameliorates

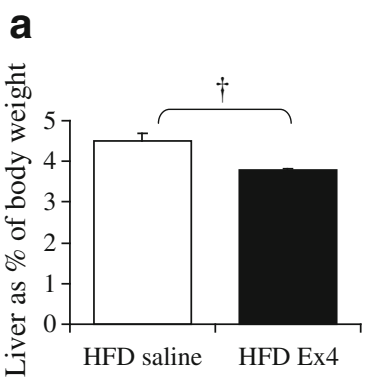

Fig. 3 Liver characteristics after 4 weeks of exendin 4 treatment on a HFD. a Liver weight expressed as a per cent of body weight, (b) triacylglycerol as $\mathrm{mg}$ per $\mathrm{g}$ of liver and (c) triacylglycerol as $\mathrm{mg}$ per
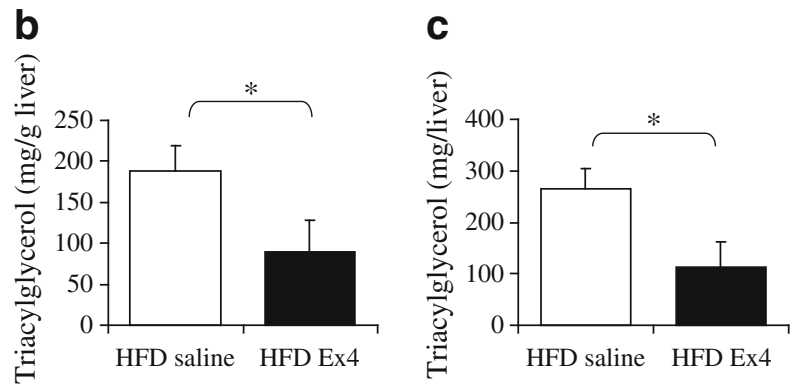

total liver weight. $n=4$ per group; ${ }^{*} p<0.05$ one-tailed $t$ test, ${ }^{\dagger} p<0.05$ two-tailed $t$ test 

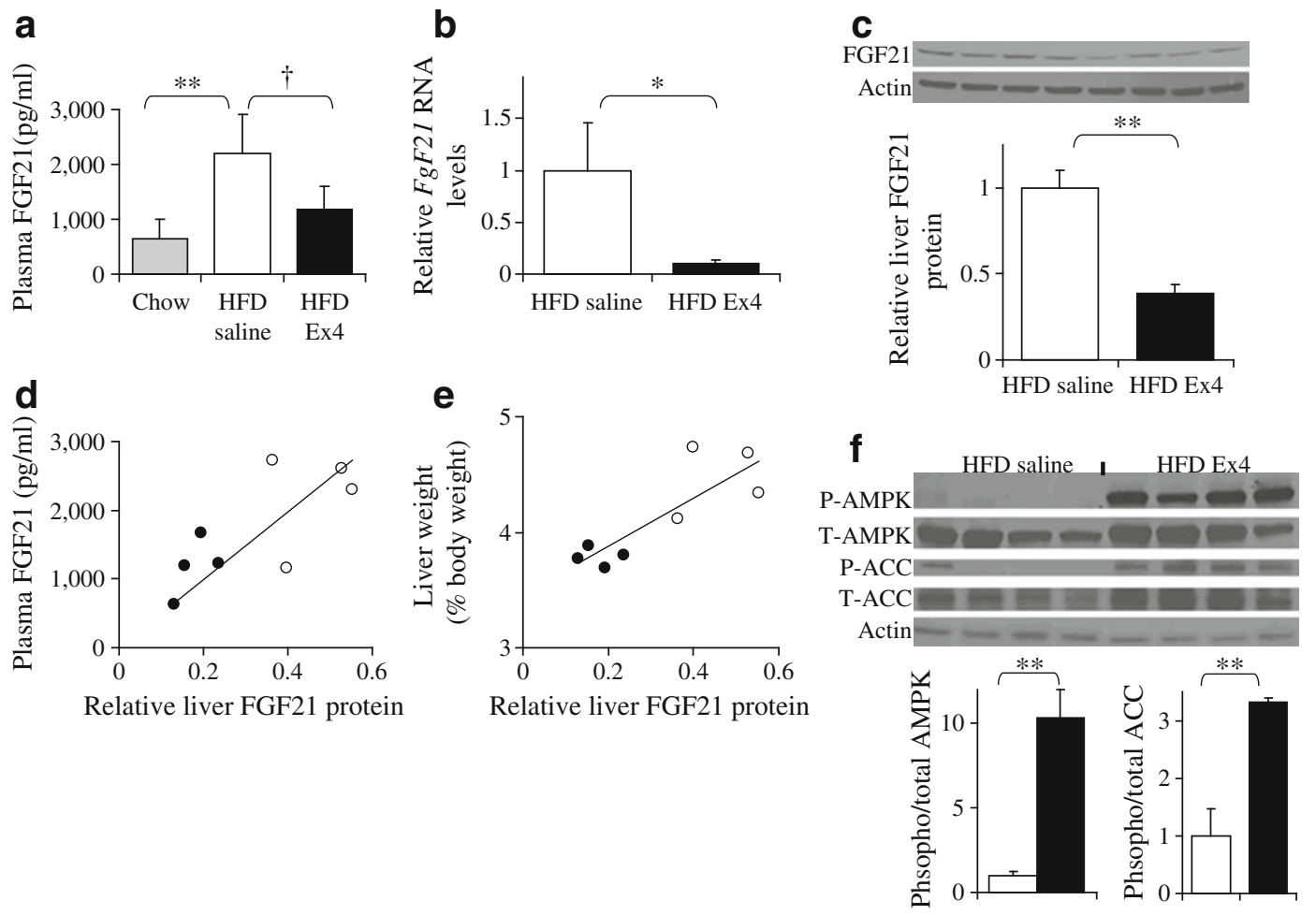

Fig. 4 FGF21 levels are altered in HFD-fed mice treated with exendin 4 (Ex4). a Plasma FGF-21 levels and (b) relative Fgf21 RNA expression in the liver measured by quantitative RT-PCR and normalised to housekeeping TATA-box binding protein. c FGF21 protein levels in the liver normalised to actin. d Correlation of liver FGF21 protein levels with plasma FGF21 (white circles, HFD saline;

hepatic FGF21 resistance. Enhanced AMPK phosphorylation was not mediated by differences in circulating adiponectin levels, since plasma adiponectin levels did not change significantly $(4.3 \pm 1.7 \mu \mathrm{g} / \mathrm{ml}$ HFD saline vs $4.9 \pm 1.4 \mu \mathrm{g} / \mathrm{ml}$ HFD exendin 4) in this mouse model.

\section{Discussion}

The results of the present study demonstrate that exenatide, a GLP1R agonist, decreases hepatic fat content and alters plasma FGF21 levels in humans with type 2 diabetes, as well as in a DIO mouse model. On its own, pioglitazone, a peroxisome proliferator-activated receptor (PPAR)- $\gamma$ agonist, does not alter plasma FGF21 levels, despite an associated decrease in hepatic fat content in patients with type 2 diabetes. However, combined exenatide and pioglitazone treatment was associated with a significant decrease in plasma FGF21 levels and a significantly greater reduction of hepatic fat compared with pioglitazone therapy. We replicated this finding in a HFD DIO, and demonstrate, for the first time, that a short period (4 weeks) of exendin 4 treatment by continuous delivery reduces black circles HFD exendin 4). e Correlation of liver FGF21 protein levels with liver weight as per cent of body weight. f Blot showing abundance of phosphorylated (P) AMPK and ACC in the liver of HFD-saline and HFD-exendin 4 mice, with quantification of AMPK and ACC. $n=4$ per group; ${ }^{*} p<0.05$ one-tailed $t$ test; ${ }^{\dagger} p<0.05,{ }^{* *} p<$ 0.01 two-tailed $t$ test

hepatic triacylglycerol content, as well as liver and plasma FGF21 levels. Hepatic AMPK phosphorylation was increased and was associated with increased ACC phosphorylation. In contrast to our previous work [14], a reduction of hepatic triacylglycerol was observed, although no significant difference in circulating adiponectin, body weight or fat mass was seen in DIO mice. Hepatic AMPK phosphorylation correlated negatively with hepatic FGF21. These results suggest that exenatide reduces hepatic FGF21 resistance in association with a reduction of hepatic triacylglycerol content in animal models of obesity, as well as in obese patients with type 2 diabetes.

Previous studies have reported regulation of FGF21 by PPAR- $\alpha$, but not PPAR- $\gamma$ agonists in humans. There was no effect on FGF21 levels following rosiglitazone (PPAR- $\gamma$ agonist) treatment in non-diabetic participants [19]. We did not observe a significant change in plasma FGF21 levels following 12 months of pioglitazone treatment. Treatment with PPAR $-\gamma$ agonists is associated with a reduction of plasma NEFA levels and NEFA turnover, and a shift in fat distribution from visceral and hepatic to subcutaneous depots, with improved hepatic and peripheral (muscle) insulin sensitivity, in spite of an increase in body weight 
$[16,17,20]$. Our findings suggest that reductions in hepatic steatosis as such do not result in a decline in FGF21 levels and an improvement in FGF21 resistance in pioglitazonetreated type 2 diabetic patients. Plasma FGF21 levels correlate with BMI in humans [9]. The reduction in hepatic fat content following pioglitazone therapy could therefore be associated with overall weight gain, abrogating any positive effect of the drug on circulating FGF21 levels. Exenatide, when combined with pioglitazone, acted more as an appetite suppressant, leading to no change in body weight at 12 months and thereby allowing a reduction of plasma FGF21 levels, as well as a greater decrease in hepatic fat content. However, this interpretation partially assumes that the changing adipose depots are the source of FGF21. Contrary to this, Dushay et al. [10] have recently shown that the major source of circulating FGF21 appears to be the liver in humans, as they did not find FGF21 production in subcutaneous or omental human adipose tissue. Our results suggest that there is a dissociation between hepatic steotosis and plasma FGF21 levels in pioglitazone-treated type 2 diabetic patients. In addition, our present results suggest a direct effect of exenatide on hepatic FGF21 resistance in patients with type 2 diabetes.

We further examined the effects of exendin- 4 on hepatic FGF21 resistance and liver steatosis in a rodent model of obesity. We have previously examined the long-term effects (16 weeks) of elevated steady-state levels of exendin 4 provided by in vivo gene therapy with a HDAd [14]. In DIO mice, HDAd-exendin 4 treatment improved glucose homeostasis, enhanced insulin action, enhanced energy expenditure, reversed hepatic steatosis and decreased hepatic expression of genes involved in de novo fatty acid synthesis, including Accl (also known as Acaca), fatty acid synthase and stearoyl CoA desaturase 1 [14]. Consistent with these observations, we found similar reductions in hepatic fat in the present study using subcutaneous osmotic pump delivery of exendin 4 (Fig. 3). Thus exendin 4 enhanced AMPK phosphorylation even in the absence of a significant effect on adiposity (Figs 2, 4). Despite the importance of FGF21 in the regulation of glucose, lipid and energy homeostasis, the mechanisms by which FGF21 functions as a metabolic regulator remain largely unknown. Recent studies suggest that FGF21 may regulate fat metabolism by several mechanisms. Chau et al. [7] have shown that FGF21 regulates energy homeostasis in adipocytes through activation of AMPK, resulting in enhanced mitochondrial oxidative function. AMPK phosphorylation levels were increased by FGF21 treatment in adipocytes as well as in WAT from $o b / o b$ mice. We observed an inverse relationship between hepatic AMPK phosphorylation and hepatic FGF21 protein in our exendin 4-treated mice $(r=-0.865$, $p=0.006$ ), suggesting an amelioration of hepatic FGF21 resistance. More recently, Fisher et al. [21] examined the effects of FGF21 on the liver in vivo, and administered FGF21 to mice to analyse acute effects on signalling and gene expression. They showed that FGF21 acts directly on the liver to stimulate phosphorylation of FGFR substrate 2 and extracellular signal-regulated kinase $1 / 2$. Acute FGF21 treatment induced hepatic production of key regulators of glucose and lipid metabolism by PPAR- $\gamma$ coactivator (PGC-1 $\alpha$ )-dependent as well as PGC-1-independent mechanisms. The precise molecular mechanism(s) responsible for exendin 4-induced improvements in hepatic FGF21 resistance in DIO needs to be examined in future studies.

We have shown that in type 2 diabetic patients treated with pioglitazone and exenatide for 12 months the increase in circulating adiponectin levels was greater than in patients with addition of pioglitazone alone [15]. Similarly, our previous work had demonstrated that 16 weeks of HDAdexendin 4 in DIO mice was associated with a significant increase in plasma adiponectin levels [14]. However, a shorter duration of exendin 4 treatment (4 weeks) in the present study was not associated with a significant change in circulating adiponectin levels. It is noteworthy that 4 weeks of exendin 4 treatment in DIO mice reduced hepatic steatosis and enhanced AMPK phosphorylation, even in the absence of significant alterations in body weight or circulating adiponectin levels

FGF21 enhances hepatic and adipocyte insulin sensitivity in animals [2-6]. However, studies in obese and type 2 diabetic participants have demonstrated elevated levels of FGF21 in the presence of hepatic and peripheral insulin resistance, and a correlation between insulin resistance and plasma FGF21 levels [9]. It has been unclear from these human studies whether this increase in circulating FGF21 represents a compensatory increase in response to insulin resistance or whether the increased levels play a causative role in the pathogenesis of insulin resistance. Recent studies in obese animals and patients with NAFLD suggest that hepatic FGF21 resistance is the more likely possibility $[8,10]$. Although we did not treat lean non-diabetic humans with exenatide, it would be interesting to examine whether exenatide reduces plasma FGF21 levels in the absence of FGF21 resistance and NAFLD.

In conclusion, we have shown that exenatide treatment in type 2 diabetic patients and a DIO is associated with a decrease in FGF21 and hepatic fat, and an increase in hepatic AMPK and ACC phosphorylation, possibly a sign of improved FGF21 resistance in the liver. Future studies on FGF21 as a potential therapeutic target in the treatment of type 2 diabetes and NAFLD may necessitate examining the effects of combined exenatide and FGF21 treatment on hepatic fat and glucose metabolism. 
Acknowledgements This work was supported in parts by a research grant from the American Diabetes Association Clinical Research Award, the Ron MacDonald Foundation at St Luke's Episcopal Hospital, Amylin Pharmaceuticals and Eli-Lilly (to M. Bajaj). The sponsors had no role in the study design or in the collection, analysis or interpretation of the data or manuscript writing. S. Samson was supported by a Molecular Medicine Scholars Training Grant (NIH T-32 HL-66991) and an American Diabetes Association Junior Faculty Award. Additional support was from HL-51586 (to L. Chan) and the Diabetes and Endocrinology Research Center DERC (P30DK079638). L. Chan is also supported by the Rutherford Chair for Diabetes Research from St Luke's Episcopal Hospital and by the T.T. and W.F. Chao Foundation. E.V. Gonzalez was supported by an American Diabetes Association Minority Mentor-based Fellowship.

Contribution statement SS contributed to the study design, acquisition of data, data interpretation and analysis, and manuscript writing. PS contributed to the acquisition of data, data analysis and manuscript writing. MJ contributed to the recruitment of patients, the acquisition of data, collection of patient samples, data analysis and revision of the manuscript. EVG contributed to study design, performance of the animal studies, the acquisition and analysis of data and manuscript writing. AG contributed to the data analysis and revision of the manuscript. RK contributed to MRS measurements, data analysis and manuscript writing. RM contributed to MRS measurements, data analysis and manuscript writing. LC contributed to study design, funding, supervision of the animal studies, data interpretation and analysis, and manuscript writing. MB contributed to study design, funding, the acquisition of data, supervision of the study, data interpretation and analysis, and writing of the manuscript. All authors approved the final version of the paper for publication.

Duality of interest M. Bajaj has received research grant support from Takeda Pharmaceuticals, Eli-Lilly and Amylin. He consults for Takeda Pharmaceuticals, and has received lecture fees from Takeda Pharmaceuticals, Merck, Eli-Lilly, Boehringer Ingelheim and sanofiaventis. S. Samson, P. Sathyanarayana, M. Jogi, E. Gonzalez, A. Gutierrez, R. Muthupillai, R. Krishnamurthy and L. Chan declare that for them there is no duality of interest associated with this manuscript.

\section{References}

1. Itoh N (2010) Hormone-like (endocrine) Fgfs: their evolutionary history and roles in development, metabolism, and disease. Cell Tissue Res 342:1-11

2. Murata Y, Konishi M, Itoh N (2011) FGF21 as an endocrine regulator in lipid metabolism: from molecular evolution to physiology and pathophysiology. J Nutr Metab 2011:981315

3. Kharitonenkov A, Shanafelt AB (2009) FGF21: a novel prospect for the treatment of metabolic diseases. Curr Opin Investig Drugs 10:359-364

4. Kharitonenkov A, Larsen P (2011) FGF21 reloaded: challenges of a rapidly growing field. Trends Endocrinol Metab 22:81-86
5. Kharitonenkov A, Shiyanova TL, Koester A et al (2005) FGF-21 as a novel metabolic regulator. J Clin Invest 115:1627-1635

6. Xu J, Lloyd DJ, Hale C et al (2009) Fibroblast growth factor 21 reverses hepatic steatosis, increases energy expenditure, and improves insulin sensitivity in diet-induced obese mice. Diabetes 58:250-259

7. Chau MD, Gao J, Yang Q et al (2010) Fibroblast growth factor 21 regulates energy metabolism by activating the AMPK-SIRT1PGC-1alpha pathway. Proc Natl Acad Sci USA 107:12553-12558

8. Fisher FM, Chui PC, Antonellis PJ et al (2010) Obesity is a fibroblast growth factor 21 (FGF21)-resistant state. Diabetes 59:2781-2789

9. Chavez AO, Molina-Carrion M, Abdul-Ghani MA et al (2009) Circulating fibroblast growth factor-21 is elevated in impaired glucose tolerance and type 2 diabetes and correlates with muscle and hepatic insulin resistance. Diabetes Care 32:1542-1546

10. Dushay J, Chui PC, Gopalakrishnan GS et al (2010) Increased fibroblast growth factor 21 in obesity and nonalcoholic fatty liver disease. Gastroenterology 139:456-463

11. Gutzwiller JP, Drewe J, Goke B et al (1999) Glucagon-like peptide-1 promotes satiety and reduces food intake in patients with diabetes mellitus type 2. Am J Physiol 276:R1541-R1544

12. Toft-Nielsen MB, Madsbad S, Holst J (1999) Continuous subcutaneous infusion of glucagon-like peptide 1 lowers plasma glucose and reduces appetite in type 2 diabetic patients. Diabetes Care 22:1137-1143

13. Ding XK, Saxena NK, Lin SB et al (2006) Exendin-4, a glucagonlike protein-1 (GLP-1) receptor agonist, reverses hepatic steatosis in ob/ob mice. Hepatology 43:173-181

14. Samson SL, Gonzalez EV, Yechoor V et al (2008) Gene therapy for diabetes: metabolic effects of helper-dependent adenoviral exendin 4 expression in a diet-induced obesity mouse model. Mol Ther 16:1805-1812

15. Sathyanarayana P, Jogi M, Muthupillai R et al (2011) Effects of combined exenatide and pioglitazone therapy on hepatic fat content in type 2 diabetes. Obesity. doi:10.1038/oby.2011.152

16. Bajaj M, Suraamornkul S, Piper P et al (2004) Decreased plasma adiponectin concentrations are closely related to hepatic fat content and hepatic insulin resistance in pioglitazone-treated type 2 diabetic patients. J Clin Endocrinol Metab 89:200-206

17. Bajaj M, Suraamornkul S, Pratipanawatr T et al (2003) Pioglitazone reduces hepatic fat content and augments splanchnic glucose uptake in patients with type 2 diabetes. Diabetes 52:1364 1370

18. Bligh EG, Dyer WJ (1959) A rapid method of total lipid extraction and purification. Can J Biochem Physiol 37:911917

19. Christodoulides C, Dyson P, Sprecher D et al (2009) Circulating fibroblast growth factor 21 is induced by peroxisome proliferatoractivated receptor agonists but not ketosis in man. J Clin Endocrinol Metab 94:3594-3601

20. Mayerson AB, Hundal RS, Dufour S et al (2002) The effects of rosiglitazone on insulin sensitivity, lipolysis, and hepatic and skeletal muscle triglyceride content in patients with type 2 diabetes. Diabetes 51:797-802

21. Fisher FM, Estall JL, Adams AC et al (2011) Integrated regulation of hepatic metabolism by fibroblast growth factor 21 (FGF21) in vivo. Endocrinology 152:2996-3004 